and to that of the early Near and Middle East has still to be determined, even though discovery is rapidly making it more clear almost day by day.

As the result of an analysis of the archæological, linguistic and ethnological evidence, Dr. Hutton arrives at the conclusion that the Indus civilisation is to be assigned to a Dravidian-speaking branch of the Mediterranean race, with possibly some Armenoid admixture, and in an acute study of Hinduism in which he assigns its contribution in that composite whole to each of the racial elements in the Hindu peoples, the prepotent and most characteristic features are assigned to the Mediterranean rather than the Aryan. It is significant, however, that he regards this whole as having been 'built up' locally.

We cannot enter now upon a consideration of the scientific bases of Dr. Hutton's conclusions. There can be no question, however, that discussion of his views hereafter will not be spared. In their practical aspect, which in India, where religion is the unifying political influence, is never far away, it may be said that the bearing of his theories is summed up in this sentence, in which he states: "it is not difficult to understand the claim of certain politicians that the term Hinduism should cover all religions having their origin in India, even though we hold that the original impulse came from the Mediterranean or Asia Minor".

\section{The 24-Hour System of Time Reckoning}

THE present method of counting longitudes east and west from a prime or zero meridian passing through the centre of the transit instrument at the Observatory of Greenwich, was decided upon at a conference held in Washington in October 1884 at which twenty-five countries were represented. The adoption, by international agreement, of a prime meridian passing through Greenwich, made possible the standard or zone system of reckoning time for civil purposes, which almost every country has adopted.

The Washington Conference in 1884 made other recommendations which did not secure such general acceptance. It expressed the hope that as soon as might be practicable, the astronomical and nautical days would be arranged everywhere to begin at mean midnight. This recommendation met with much opposition from astronomers, particularly from Prof. Simon Newcomb, whose name carried great weight. It was not until after the War that it was possible to secure agreement to this change, which was introduced at the beginning of 1925 ; the principal national ephemerides for the year 1925 and subsequent years have used an astronomical day beginning at mean midnight. A third recommendation of the Conference was in favour of counting the hours of the day from zero up to twenty-four hours. This system of counting, which avoids the necessity for 'a.m.' and 'p.m.', and the ambiguity which results if these are omitted, has been adopted in railway time-tables and for other purposes in all Continental countries, in Canada, the United States, India and elsewhere, but it has never come into general use in Great Britain.

The 24-hour system of reckoning time has been permanently adopted in Great Britain by the Army, Navy and Air Force. It is not without significance that the three defence departments of the country have all adopted the system which eliminates the possibility of errors likely to arise when the hours are counted from 1 to 12 twice a day. The advisability of adopting the 24-hour system for official and other purposes was considered in 1919 by a Home Office Committee, presided over by Lord Stonehaven. The Committee went into the matter thoroughly, found no objection to it and unanimously recommended its adoption by the Post Office and railway companies. But although no legislative action was required, no steps were taken to adopt the recommendation. The matter has been raised on a number of occasions in the House of Lords without definite result. It will come up again for discussion on December 7, when a motion, to be introduced by Lord Newton, urging the adoption of the recommendations of the Stonehaven Committee, will be discussed.

On various occasions there has been correspondence in the Times and other daily papers on the subject of this reform. Opinion in favour of the change has largely preponderated, and it has been significant that those who have opposed it have been those having no experience of it, whilst those who have had experience of it have strongly favoured it. The railway companies intimated in 1931 and again in 1932 that they were prepared to adopt the 24 -hour system if the Government would give the lead. When the matter was discussed in the House of Lords in 1932, the Earl of Lucan, speaking as the representative of the Home Office, stated that "The view of the Government is that so far the public has not shown that it wants the change". A weaker argument could not have been put forward, 
but this was the strongest argument against the reform that could be found.

The 24-hour system can be used along with the present system. For normal social and business purposes, there is little possibility of confusing a.m. and p.m.; nor is any alteration of clocks, by having a 24-hour dial or by having two sets of figures from 1 to 12 and 13 to 24 , necessary. If the recommendations of the Stonehaven Committee are adopted, the 24-hour system would be used by the Post Office for timing letters and telegrams, on pillar boxes, etc., and the railway companies would use it in time-tables, and so avoid the necessity of different types for times before and after noon. The public would soon become familiar with the system and recognise its convenience. We strongly hope that the House of Lords will approve the motion to be introduced by Lord Newton on December 7, and that the Government will not delay in giving effect to the recommendation.

\section{Chain Reactions}

(1) Kettenreaktionen. Von Klaus Clusius. (Fortschritte der Chemie, Physik und physikalischen Chemie, herausgegeben von Prof. Dr. A. Eucken, Band 21, Heft 5.) Pp. iv +73 . (Berlin : Gebrüder Borntraeger, 1932.) 12.80 gold marks.

(2) The Kinetics of Chemical Change in Gaseous Systems. By C. N. Hinshelwood. Third edition. Pp. vii +392 . (Oxford: Clarendon Press; London: Oxford University Press, 1933.) 15s. net.

\HE theory of chain reactions was introduced in order to account for the abnormally large yields which are often found in photochemical reactions, when compared with Einstein's 'Law of Photochemical Equivalence', according to which each quantum of absorbed light activates or decomposes one molecule of the absorbing medium. Thus Bodenstein in 1913 found that, in mixtures of hydrogen and chlorine, $10^{5}$ molecules were decomposed by each quantum instead of only one. In order to maintain the validity of Einstein's law, he therefore postulated that a secondary action took place, in which the primary product was regenerated again and again, so that an indefinitely large vield of the product could be obtained. For the special case of hydrogen and chlorine, Nernst in 1918 postulated the following mechanism:

I. Primary reaction: $\mathrm{Cl}_{2}+h_{\nu}=2 \mathrm{Cl}$.
II. Secondary reactions (reaction chain) :

$$
\begin{gathered}
\mathrm{H}_{2}+\mathrm{Cl}=\mathrm{HCl}+\mathrm{H}, \mathrm{H}+\mathrm{Cl}_{2}= \\
\mathrm{HCl}+\mathrm{Cl}, \mathrm{H}_{2}+\mathrm{Cl}=\text { etc. }
\end{gathered}
$$

III. End of reaction chain :

$$
2 \mathrm{Cl}=\mathrm{Cl}_{2} ; 2 \mathrm{H}=\mathrm{H}_{2} .
$$

In this scheme, atoms of chlorine are the primary products of the photochemical action. Since atoms of hydrogen are not present in the system, the conventional double decomposition shown by the equation $\mathrm{H}_{2}+\mathrm{Cl}_{2}=[2 \mathrm{H}+2 \mathrm{Cl}]=2 \mathrm{HCl}$ cannot take place and the action can only proceed by the action of atoms of chlorine on molecules of hydrogen. This action, however, leads to the liberation of an atom of hydrogen for each atom of chlorine that is used, $\mathrm{Cl}+\mathrm{H}_{2}=\mathrm{HCl}+\mathrm{H}$. This atom of hydrogen in its turn can only interact with a molecule of chlorine, since atoms of chlorine are present only in minimal concentrations, and thus liberates another atom of chlorine, $\mathrm{H}+\mathrm{Cl}_{2}=$ $\mathrm{HCl}+\mathrm{Cl}$. The primary product of the photochemical change has thus been reproduced and the cycle can be repeated indefinitely as a chain reaction. If, however, two atoms should happen to collide, instead of one atom and one molecule, the interaction (in presence of a 'third body' to carry away the energy of combination) would not give rise to a free atom, and the chain would therefore be broken.

Chain reactions can be initiated by the action of $\alpha$-particles or of swift electrons, as well as by the action of light; or the 'carrier' may be produced by an ordinary chemical reaction, as in Polanyi's experiments, in which atoms of chlorine were produced by the action of dilute sodium vapour on chlorine gas, $\mathrm{Na}+\mathrm{Cl}_{\mathbf{2}}=\mathrm{NaCl}+\mathrm{Cl}$. The free atoms or radicals thus produced are characteristic of 'material chains', but 'energy chains' are also possible, in which an activated molecule hands on its energy to another molecule. The life of these excited molecules is so much shorter than that of free atoms or radicals that 'energy chains' are likely to be much less frequent than 'material chains'; but the latter normally include also a transfer of energy, since the initiation of a chain depends on creating a centre of high activity, for example, by bringing about under the influence of light an endothermic dissociation with a high heat of activation; and this energy is handed on from one atom to another in the chain. The secondary reactions, on the other hand, must have a very low energy of activation, in order that the chain may be propagated easily, and must 